\title{
ARTICLE
}

Received 4 Apr 2014 | Accepted 3 Jul 2014 | Published 11 Aug $2014 \quad$ DOl: 10.1038/ncomms5587

\section{Tension on the linker gates the ATP-dependent release of dynein from microtubules}

Frank B. Cleary¹, Mark A. Dewitt ${ }^{1}$, Thomas Bilyard², Zaw Min Htet ${ }^{2}$, Vladislav Belyy ${ }^{1}$, Danna D. Chan², Amy Y. Chang ${ }^{2} \&$ Ahmet Yildiz ${ }^{2}$

Cytoplasmic dynein is a dimeric motor that transports intracellular cargoes towards the minus end of microtubules (MTs). In contrast to other processive motors, stepping of the dynein motor domains (heads) is not precisely coordinated. Therefore, the mechanism of dynein processivity remains unclear. Here, by engineering the mechanical and catalytic properties of the motor, we show that dynein processivity minimally requires a single active head and a second inert MT-binding domain. Processivity arises from a high ratio of MT-bound to unbound time, and not from interhead communication. In addition, nucleotide-dependent microtubule release is gated by tension on the linker domain. Intramolecular tension sensing is observed in dynein's stepping motion at high interhead separations. On the basis of these results, we propose a quantitative model for the stepping characteristics of dynein and its response to chemical and mechanical perturbation.

\footnotetext{
${ }^{1}$ Biophysics Graduate Group, University of California, Berkeley, California 94720, USA. ${ }^{2}$ Department of Physics, University of California, Berkeley, California 94720, USA. Correspondence and requests for materials should be addressed to A.Y. (email: yildiz@berkeley.edu).
} 
C ytoplasmic dynein is responsible for nearly all microtubule (MT) minus-end-directed transport in eukaryotes ${ }^{1}$. In interphase cells, dynein transports membrane-bound organelles and vesicles ${ }^{1}$. During mitosis, dynein positions the spindle $^{2}$, focuses the MTs into poles ${ }^{3}$ and regulates the spindle assembly checkpoint ${ }^{4}$. Impaired dynein function has been implicated in motor neuron degeneration ${ }^{5}$, lissencephaly, and primary ciliary dyskinesia ${ }^{6}$. The ability of dynein to move processively and maintain contact with the MT is thought to be essential for its cellular roles, and small perturbations in dynein processivity were implicated in motor neuron degeneration ${ }^{7}$. However, the mechanism underlying these properties is not well understood.

Dynein's structure is unique among cytoskeletal motors. The $\sim 350 \mathrm{kDa}$ dynein motor domain (head) is much larger than that of kinesin or myosin, and contains six AAA + subunits arranged in a hexameric ring (Fig. 1a) ${ }^{7-9}$. Unlike kinesin, whose MT binding and ATPase sites are part of the same globular domain,

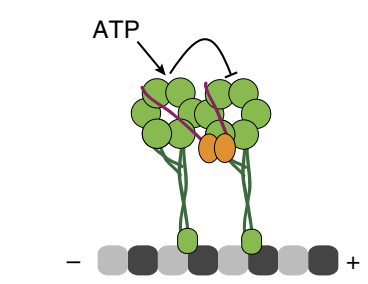

c
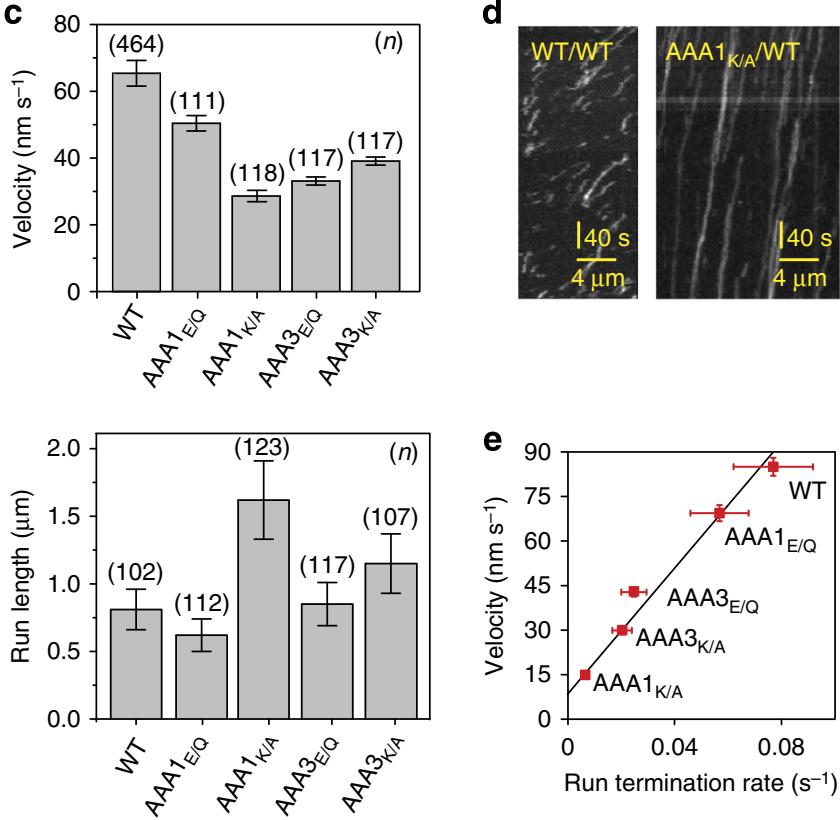

Figure 1 | Motile properties of heterodimeric dynein constructs bearing a single ATPase mutation. (a) Dynein monomers truncated at the $\mathrm{N}$-terminal tail are dimerized through an $\mathrm{N}$-terminal GST tag (orange). The nucleotide-gating model requires the ATPase cycles of the two heads to be kept out of phase. (b) A schematic (right) of FRB-FKBP12 dynein heterodimer on a MT. One of the heads (blue) contains specific mutations at an ATPase site. (c) Average velocity ( \pm s.e.m.) and run length ( $\pm 95 \%$ $\mathrm{Cl}$ ) of TMR-labelled dynein motors at $2 \mathrm{mM}$ ATP. One WT monomer is dimerized with a partner carrying the indicated ATPase mutation. (d) Kymographs showing the processive movement of WT/WT and $\mathrm{AAA} 1_{\mathrm{K} / \mathrm{A}} / \mathrm{WT}$ motors. (e) Plot of velocity versus run termination rate for the indicated heterodimers shows a linear relationship (black line). dynein's MT-binding domain (MTBD) is located at the end of a coiled-coil stalk ${ }^{10}$. The linker, a helical bundle extending from the ring, is thought to be the mechanical element of dynein motility $9,11,12$. The linker shifts its position relative to the ring as a function of the nucleotide state of AAA1 (ref. 12) and its conformation correlates with MT affinity ${ }^{13}$. The linker makes multiple contacts with the ring, and disruption of these contacts reduces catalytic activity ${ }^{7,8}$. Therefore, apart from functioning as a lever arm, the linker may also play a regulatory role in the nucleotide hydrolysis cycle.

The mechanism of dynein motility also differs significantly from other motors. Processive kinesin and myosins walk handover-hand $^{14-17}$, in which the heads are coordinated and take alternating steps. In contrast, dynein heads step independently of their partner ${ }^{18,19}$. It remains unknown what prevents both heads from releasing simultaneously in the absence of strict interhead coordination. Established views of motor processivity propose that, to keep the two heads out of phase, a chemical or structural change in one head must be gated until the other head proceeds through a critical step in its cycle. Several mechanisms have been proposed for motor gating 20 . When two heads are bound to the MT, the motor may experience tension through the linker domains. Interhead tension may accelerate the release of the rear head or inhibit nucleotide binding to the front head ${ }^{21,22}$. Reducing tension through peptide insertions affects kinesin velocity and efficiency of converting ATP hydrolysis into motion $^{23,24}$, and decreases myosin VI processivity ${ }^{25}$. Alternatively, the nucleotide cycle of one head may allosterically affect that of the other head, so one head stays tightly bound to MT until the other head detaches from the MT and takes a step ${ }^{26}$. In the case of $\mathrm{AAA}+$ enzymes, the rings may also directly interact with each other ${ }^{27}$. Indeed, stacking interactions between the rings were observed in electron microscopy images of axonemal dynein ${ }^{28}$.

In this study, we tested the structural and catalytic requirements of dynein processivity. To construct a minimally processive dynein motor, we altered the mechanical and catalytic properties of Saccharomyces cerevisiae dynein and tested their role in motility at a single molecule level. We find that processivity minimally requires a single active monomer and a tether retaining only the MTBD. The degree of processivity is determined by the MT affinity of the tether. Dynein processivity does not require any of these gating strategies to prevent simultaneous dissociation of both heads from MT. However, dynein remains partially gated at high interhead separations. To test whether the mechanism of this conditional gating is due to linker tension, we pulled dynein monomers through their $\mathrm{C}$ terminus or the $\mathrm{N}$-terminal linker using an optical trap under different nucleotide conditions. Remarkably, exerting tension on the linker fully abolishes the ATP-dependent MT release of the motor. High-resolution multicolour tracking of a heterodimeric dynein with one active and one inactive head showed that the stepping rate of an active head is high when it is positioned close to its inactive partner, but remains low when it is positioned apart. The results reveal a critical role of the linker in gating dynein stepping behaviour and suggest that dynein steps in a tension-dependent manner at high interhead separations. Using our experimental results, we developed a computational model for the dynein stepping mechanism in the absence of tight interhead communication.

\section{Results}

Does dynein processivity require nucleotide gating? A nucleotide-gating mechanism proposes that the nucleotide states of the heads remain out of phase such that one head remains 
tightly bound to the MT as the other head releases and takes a step. The model requires both heads to be catalytically active and able to change their MT affinity in a nucleotide-dependent manner (Fig. 1a). To test the predictions of this model, we disrupted ATP binding (K/A mutation in the Walker A motif) or hydrolysis (E/Q mutation in the Walker B motif) in specific $\mathrm{AAA}+$ domains. Studies on dynein homodimers showed that ATPase mutations of monomers of a truncated $331-\mathrm{kDa}$ S. cerevisiae dynein (herein referred to as Dyn) at AAA1 fully abolish motility $^{29,30}$ and AAA3 mutations result in movement tenfold slower than native dynein ${ }^{29,30}$. We previously demonstrated that a heterodimer of $\mathrm{AAAl}_{\mathrm{E} / \mathrm{Q}}(\mathrm{E} 1849 \mathrm{Q})$ and wild-type (WT) monomers walk processively ${ }^{18}$. However, it remained unclear whether the processive motility is facilitated by the ability of the WT monomer to drag its inactive partner, or $A A A 1_{E / Q}$ releases from MT through ATP binding to its AAA1 site. To address this question, we introduced different ATPase mutations to one of the heads of a dynein heterodimer constructed with FRB-FKBP12 tags (Fig. 1b; Supplementary Table 1) ) $^{31,32}$ and tested their motility by total internal reflection fluorescence microscopy.

A heterodimer of FRB-Dyn and FKBP12-Dyn (WT/WT) moves at slightly reduced velocity $\left(65 \pm 2 \mathrm{~nm} \mathrm{~s}^{-1}\right.$, s.e.m.) and processivity $(0.8 \pm 0.2 \mu \mathrm{m}, 95 \%$ confidence interval (CI)) compared with glutathione s-transferase (GST)-Dyn $\left(99 \pm 2 \mathrm{~nm} \mathrm{~s}^{-1}\right.$ and $1.1 \pm 0.1 \mu \mathrm{m}$, Fig. 1c), as previously shown ${ }^{32}$. When ATPase mutations were introduced to one of the monomers, both the velocity and processivity of the heterodimer were affected. Compared with WT/WT, velocity and run length of the $\mathrm{AAA} 1_{\mathrm{E} / \mathrm{Q}} / \mathrm{WT}$ heterodimer was reduced $20 \%$, while $\mathrm{AAA} 1_{\mathrm{K} / \mathrm{A}} /$ WT motors moved $60 \%$ slower and had twofold longer run length (Fig. 1c,d). These results indicate that a WT monomer can pull an inactive AAA1 mutant head faster when the mutant head has a reduced MT affinity, at the expense of reduced processivity. $\mathrm{AAA}_{\mathrm{K} / \mathrm{A}} / \mathrm{WT}$ (K2424A) and $\mathrm{AAA}_{\mathrm{E} / \mathrm{Q}} / \mathrm{WT}$ (E2488Q) heterodimers showed a similar reduction in motor velocity to $\mathrm{AAA1}_{\mathrm{K} / \mathrm{A}} / \mathrm{WT}$ (Fig. 1c), suggesting that ATPase activity at AAA3 may regulate the communication between AAAl and MTbinding affinity ${ }^{33}$

The velocity of the mutant heterodimers is linearly related to the probability of a processive run ending per unit time (Fig. 1e). The WT head can pull its inactive partner faster when it has a reduced MT affinity (for example, $A A A 1_{\mathrm{E} / \mathrm{Q}}$ ), but these constructs are less processive than those where the mutant head is locked in a state with high affinity to MT. The results suggest that the magnitude of dynein processivity is determined by the duty ratio (the ratio of MT-bound to unbound time in a stepping cycle) of each head, not by the precise coordination of the stepping cycles of the heads through a nucleotide-gating mechanism ${ }^{19,20}$.

Minimal requirements of dynein processivity. To test whether stacking interactions between the AAA + rings $^{28}$ are required for dynein motility, we created a 'ringless' dynein monomer in which the AAA + ring and the linker are replaced with a monomeric seryl-tRNA synthetase (SRS) fused with the dynein stalk and $\mathrm{MTBD}^{34,35}$. If interaction between the two rings in a dimer must occur at some point in the ATPase cycle, a dynein heterodimer with a single ATPase ring is not expected to move processively. We tested two strongly bound SRS chimeras, one with a fulllength dynein-coiled coil $\left(\mathrm{SRS}_{85: 82}\right)$ and a one-quarter-length mutant (SRS $22: 19$ ) (Fig. 2a). Both constructs showed processive, minus-end-directed motion when dimerized to the linker domain of a WT dynein monomer (Fig. 2b and Supplementary Movie 1). Remarkably, $\mathrm{SRS}_{22: 19} / \mathrm{WT}$ had a mean velocity and processivity similar to WT/WT, although it lacks the entire AAA + ring and the linker domain in one head. $\mathrm{SRS}_{85: 82} / \mathrm{WT}$ was approximately threefold slower and twofold more processive (Fig. $2 \mathrm{c}$ and Supplementary Fig. 1). The difference between the velocities and run lengths of the two constructs may be due to the geometrical constraints imposed by the altered stalk length. High-resolution tracking assays with $\mathrm{SRS}_{85: 82} / \mathrm{WT}$ (Fig. 2d) showed that $\mathrm{SRS}_{85: 82}$ had similar step-size distribution to the WT dynein monomer (Fig. 2e $)^{18}$. The results exclude direct mechanical or allosteric interactions between the two $\mathrm{AAA}+$ rings as a required mechanism for dynein processivity.

We hypothesized that the velocity and processivity of the SRS constructs depend on the MT affinity of the SRS-MTBD tether. To test whether a ringless head must maintain a high MT affinity for processive motion, we used a $\mathrm{SRS}_{89: 82}$ construct with an altered coiled-coil registry, which has nearly two orders of magnitude lower MT affinity from $\mathrm{SRS}_{85: 82}$ (ref. 34). $\mathrm{SRS}_{89: 82} / \mathrm{WT}$ was not able to move unidirectionally, and instead diffused along MTs (Supplementary Fig. 2). The lack of processivity suggests that the inactive MT tether must have sufficient MT affinity to allow processive motion. We next tested whether the velocity of a dynein monomer with a tightly bound $\mathrm{SRS}_{85: 82}$ tether could be increased by reducing the MT affinity of the tether. Addition of up to $100 \mathrm{mM} \mathrm{KCl}$ decreased the dissociation constant $\left(K_{\mathrm{d}}\right)$ of the SRS $_{85: 82}$-MTBD from MTs by $\sim 30$-fold (Supplementary Fig. 2c). While the velocity of a WT homodimer remains unaltered by addition of $100 \mathrm{mM} \mathrm{KCl}$, the velocity of the $\mathrm{SRS}_{85: 82} / \mathrm{WT}$ increased by $50 \%$ (Supplementary Fig. 2e), providing further evidence that motor velocity increases by reducing the MT affinity of a strongly bound inactive MT tether (Fig. 1c,e).

The linker is a mechanical element that powers motility. We next tested the mechanism by which the active head can drag its inactive partner forward, presumably via forces generated by the powerstroke of its linker. Although evidence that the linker can power motility was observed in truncated monomeric dyneins ${ }^{32,36}$, the role of linker in processive motility and force generation remains unclear. The powerstroke model ${ }^{9,11}$ suggests that a head that generates force must be attached to the other head through its linker domain, either to push against the other head or to pull it forward. We tested this hypothesis by engineering a heterodimer composed of two catalytically identical but mechanically distinct monomers. The N-terminal linker of one monomer was attached to the $\mathrm{C}$ terminus of the other monomer, resulting in a heterodimer of a bound-linker head (BLH) and a free-linker head (FLH) (Fig. 3a). According to the powerstroke model, inhibiting the linker swing in the BLH would be predicted to abolish motility, while motors with an inhibited FLH would remain motile.

Motility assays revealed that the FLH/BLH heterodimer moves processively towards the minus end of MTs at $85 \%$ of the velocity of the WT/WT heterodimer (Fig. 3b and Supplementary Movie 2). Intriguingly, the run length of FLH/BLH $(2.3 \pm 0.4 \mu \mathrm{m}$; mean $\pm 95 \%$ CI) was more than double that of WT/WT (Fig. 3c). We next tested the motility of FLH/BLH heterodimers carrying a single AAA1 ATPase mutation ${ }^{12}$. AAA1 mutations to the FLH resulted in similar effects to those observed for the $\mathrm{N}$-terminally dimerized mutant heterodimers: A $\mathrm{AAAl}_{\mathrm{E} / \mathrm{Q}}$ mutation $\left(\mathrm{FLH}_{\mathrm{AAA} 1 \mathrm{E} / \mathrm{Q}} / \mathrm{BLH}\right)$ had a minimal effect on motor velocity, whereas $\mathrm{FLH}_{\mathrm{AAA} 1 \mathrm{~K} / \mathrm{A}} / \mathrm{BLH}$ motors suffered a threefold reduction in velocity (Figs $1 \mathrm{c}$ and $3 \mathrm{~d}$ ). In contrast, introducing the same AAA1 mutations to the BLH (FLH/BLH AAA1 K/A $_{\text {and }}$ FLH/ BLH $_{\text {AAA I E/Q }}$ ) fully stopped directional motility (Fig. 3d,e). While $\mathrm{FLH} / \mathrm{BLH}_{\mathrm{AAA} 1 \mathrm{~K} / \mathrm{A}}$ motors remained fixed to a single location on the MT, FLH/BLH BAA1 $_{\text {E/Q }}$ showed ATP-dependent nondirectional diffusive behaviour along MTs (Fig. 3e), consistent 
a

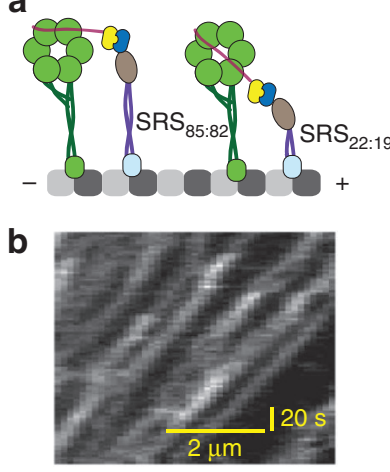

d

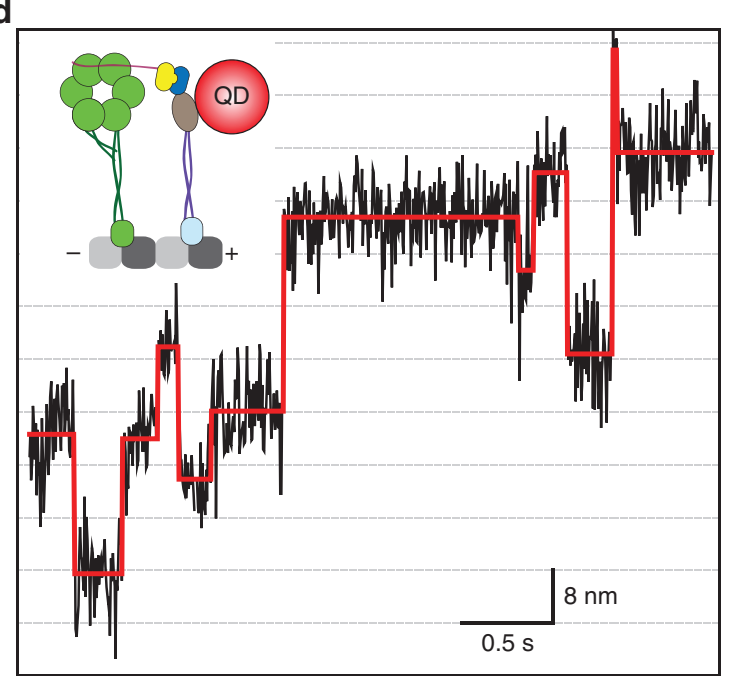

C

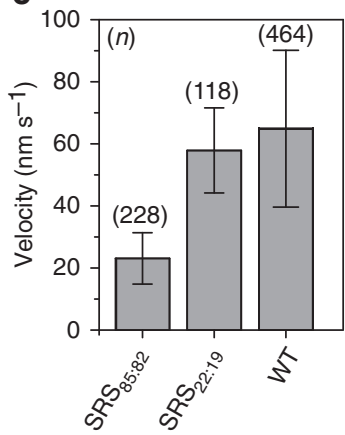

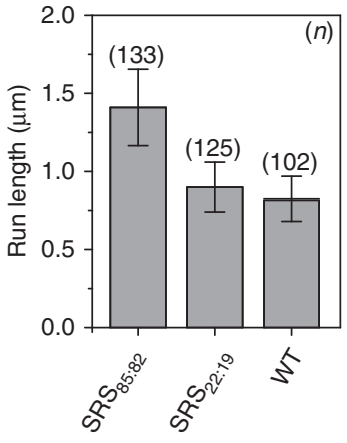

e
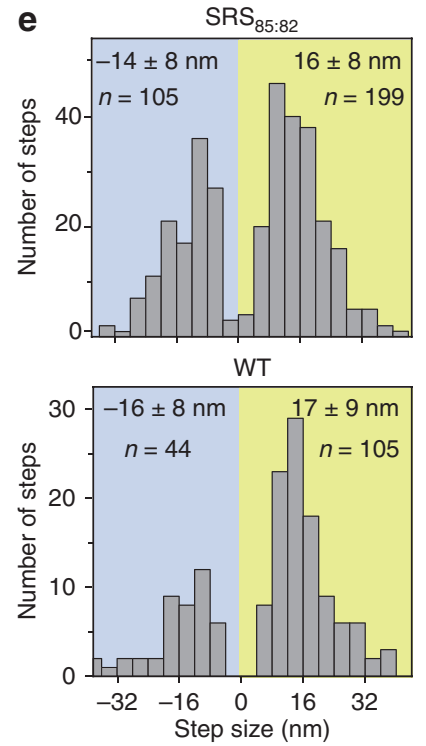

Figure 2 | Dynein processivity requires a single motor domain. (a) Possible ring-ring communication between the two heads is abolished by replacing one of the monomers with a SRS-MTBD chimera. (b) A kymograph of SRS $85: 82$ /WT showing that it moves processively along MTs. (c) The average velocity ( \pm s.d.) and run length $( \pm 95 \% \mathrm{Cl}$ ) of the SRS-MTBD/WT constructs compared with WT/WT. (d) An example high-resolution tracking trace (black) and stepping fit (red) of the SRS $85: 82$ head. (e) Histograms of the step sizes for the SRS (upper) and WT (lower) heads (mean \pm s.d.).

with reduced $\mathrm{MT}$ affinity of the $\mathrm{AAA} 1_{\mathrm{E} / \mathrm{Q}}$ monomer in the presence of ATP (Supplementary Fig. 3). The AAA3 mutants are able to undergo linker swing ${ }^{12}$, but result in slower motility ${ }^{31}$. Consistently, AAA3 mutations to FLH and BLH moved processively at lower speeds in comparison with WT constructs. Together, the results support that the ATP-dependent linker swing is the force generation mechanism for the motility of a dynein dimer.

Tension on the linker gates ATP-dependent MT release. We next investigated whether the linker domain has a regulatory role in the dynein stepping mechanism in addition to generating force. High-resolution tracking studies ${ }^{18,19}$ showed that the two heads of a dynein dimer step independently when they are positioned close to each other on a MT. However, when the heads are separated, the trailing head becomes more likely to step. At high interhead separations, dynein may experience intramolecular tension that favors the release of the trailing head $^{37}$. To investigate whether tension on the linker domain affects the mechanochemical cycle of dynein, we developed an optical trapping assay to measure the nucleotide-dependent MTrelease rates under load (Fig. 4a). Polystyrene beads were sparsely coated with monomeric dynein and moved $\pm 250 \mathrm{~nm}$ between two positions above the MT in a square-wave pattern (Fig. 4b). When a WT monomer binds to the MT, the bead is unable to follow the trap to the next position. In this state, the trap exerts a constant force on the motor, depending on the bead-trap separation, until the motor releases from the MT. The assay enables direct measurement of release rate over a large range of forces $(0.5-12 \mathrm{pN})$. A similar approach using an alternate geometry was recently used to measure myosin-unbinding forces ${ }^{38}$. Our assay is a significant improvement compared with previous motor-unbinding assays that measured average rupture force under constantly increasing force ${ }^{39}$, because it better represents the situation in a walking two-headed motor where heads are likely to experience a constant force before releasing from the MT.

We first pulled dynein monomers from the N-terminal linker domain in the absence of nucleotide. There was a clear difference in the average time a monomer remained bound to the MT, depending on the direction of the applied load. When force was applied towards the MT minus end, the monomers rapidly released from $\mathrm{MTs}$ (Fig. $5 \mathrm{c}$ ). In the apo state, the release rate increased from $5 \mathrm{~s}^{-1}$ at $1-2 \mathrm{pN}$, to $20 \mathrm{~s}^{-1}$ at $5-6 \mathrm{pN}$ (Fig. 5c). In contrast, release towards the plus end is significantly slower $\left(\sim 1.5 \mathrm{~s}^{-1}\right)$ and independent of load up to $7 \mathrm{pN}$, the highest measured stall force of native yeast dynein ${ }^{40}$. We observed a similar asymmetry in release of the $\mathrm{SRS}_{85: 82}$ chimera, which contains mouse dynein MTBD (Supplementary Fig. 3a,c). The results indicate that dynein favors release towards 
a

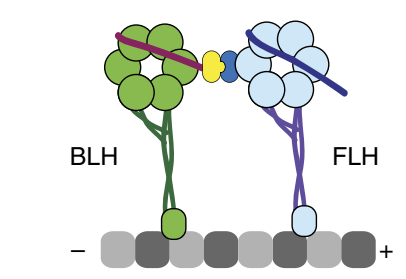

d

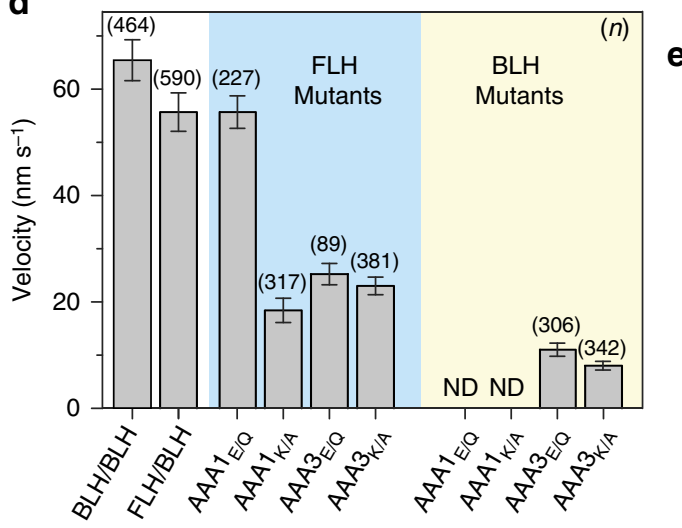

b
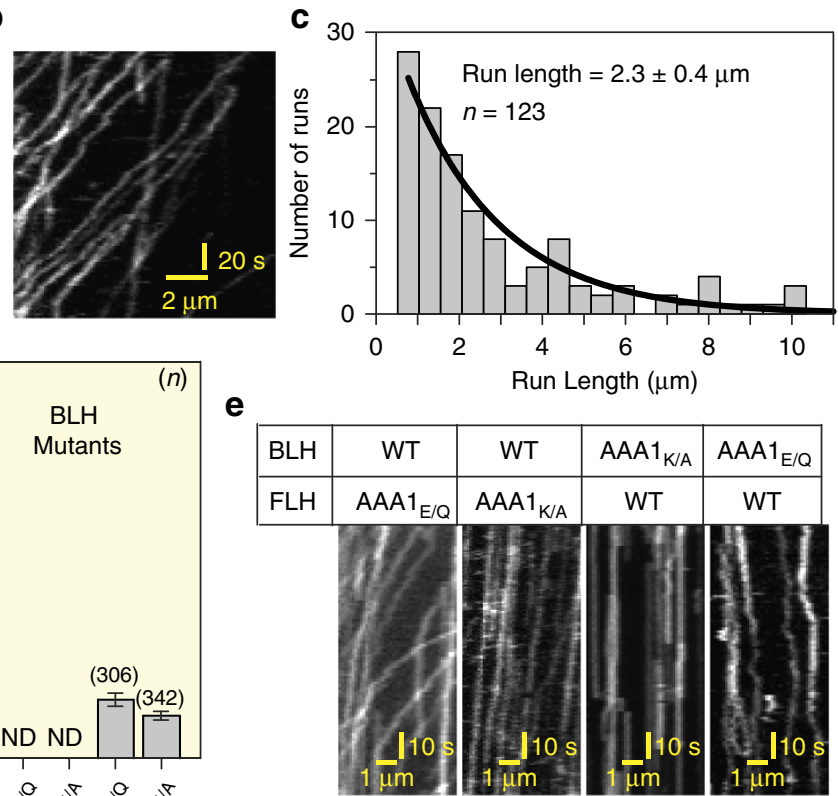

Figure 3 | The linker provides force to drive the motility of a dynein dimer. (a) Dimerization of the C-terminal ring of one head to the $\mathrm{N}$-terminal tail of the other results in a dimer of a free-linker head (FLH) and a bound-linker head (BLH). (b) Kymograph showing that the FLH/BLH heterodimer is capable of processive motility. (c) Run length histogram of FLH/BLH at $2 \mathrm{mM} \mathrm{ATP}$, with maximum likelihood fit $( \pm 95 \% \mathrm{Cl}$ ). (d) The average velocities ( \pm s.e.m.) of FLH/BLH constructs carrying an ATPase mutation in either the AAA1 or AAA3 site in one head (ND: motility not detected). (e) Kymographs of ATPase mutants of the FLH or BLH at $2 \mathrm{mM}$ ATP. The $A A A 1_{K / A}$ mutation on BLH abolishes directional motility, whereas AAA1 $/ \mathrm{E} / \mathrm{Q}$ mutation leads to nondirectional diffusion along the MT. The same mutations on FLH do not stop motility, indicating that BLH monomer is responsible for FLH/BLH motility.

its natural direction of motion under tension. The asymmetric release of a head is an intrinsic property of its stalk and MTBD, and does not depend on its AAA + ring or linker. In addition, the preferential release under forward load is not specific to yeast dynein, and may be well conserved in dyneins in higher organisms.

ATP addition is expected to increase the MT-release rate independent of load ${ }^{13}$. Surprisingly, the release rate profile was unchanged upon addition of $2 \mathrm{mM}$ ATP (Fig. 4c). We hypothesized that this could be due to the force being applied to dynein through its linker. To test this possibility, we repeated the trapping assay with the bead bound to the $\mathrm{C}$ terminus of a dynein monomer through a short (74bp) DNA tether (Fig. 4a). In the absence of ATP, we observed similar release rates to those of monomers pulled through the linker domain, implying that DNA attachment does not induce changes in the release rate. Remarkably, the release rate increased several fold upon addition of $2 \mathrm{mM}$ ATP (Fig. 4d). The results show that tension upon the linker domain inhibits nucleotide-dependent release of dynein from MTs, which may explain the altered stepping behaviour of a dynein dimer at high interhead separations ${ }^{19,20}$.

Dynein stepping is gated at high interhead separations. We next tested whether gating caused by intramolecular tension plays any role in the stepping mechanism of active dynein dimers. If tension on the linker inhibits nucleotide-dependent release from the MT, we expect to observe altered stepping rates at high interhead separations. To experimentally test this effect, we tracked the stepping behaviour of both heads of the $\mathrm{AAA} 1_{\mathrm{K} / \mathrm{A}} /$ WT heterodimer (Fig. 1b) at saturating ( $1 \mathrm{mM}$ ) ATP. The AAA $1_{\mathrm{K} / \mathrm{A}}$ mutant head could be envisioned as a MT tether in a high-affinity conformation, because it is unable to produce force ${ }^{13}$ and remains tightly bound to $\mathrm{MT}^{31}$. Therefore, AAA1 $1_{\mathrm{K} / \mathrm{A}}$ can only step when released from MT under tension generated by the linker domain of the WT head. The effects of this mutation were evident in two-colour tracking traces (Fig. 5a). The step sizes of both heads negatively correlate with on-axis separation of the heads (Fig. 5b), similar to native dynein. However, the net bias to step towards the MT minus end was significantly higher in the WT head than in the $\mathrm{AAA}_{\mathrm{K} / \mathrm{A}}$ head $(11.1 \pm 2.1$ versus $4.6 \pm 2.2 \mathrm{~nm}( \pm 95 \% \mathrm{CI})$, respectively) when the heads are positioned close to each other. The $A A A 1_{K / A}$ head trails often ( $72 \%$ of dwells) and has a low probability (28\%) to take a step when it is in the leading position (Fig. 5b,c). The results are consistent with the inability of the $A A A 1_{K / A}$ head to step on its own and the resistance of the MT-binding interface to release under plus-end-directed forces.

If the heads are fully uncoordinated, the WT head in the $\mathrm{AAA}_{\mathrm{K} / \mathrm{A}} / \mathrm{WT}$ heterodimer is expected to have the same stepping rate as a head in a WT/WT homodimer. We observed that the stepping rate of the WT head $\left(3.8 \pm 0.5 \mathrm{~s}^{-1}\right)$ is significantly higher than the mutant head $\left(2.8 \pm 0.4 \mathrm{~s}^{-1}, P<0.0001\right.$, Kolmogorov-Smirnov test, Fig. 5d), but it is lower than the MT-stimulated ATPase rate per head $\left(8 s^{-1}\right)$ in WT/WT ${ }^{32}$. This suggests that the stepping motion of the WT head is slowed by the presence of the mutant head.

The analysis of two-colour traces as a function of interhead separation revealed that the stepping of the heads depend on interhead separation. The stepping rate of the $A A A 1_{K / A}$ head was low and slightly decreasing with increased interhead separation (Fig. 5e). In comparison, the stepping rate of the WT head $\left(6.0 \pm 2.0 \mathrm{~s}^{-1}\right)$ was nearly as high as the bulk ATPase rate at low interhead separations ${ }^{29}$, but it is similar to that of $\mathrm{AAA} 1_{\mathrm{K} / \mathrm{A}}$ at high interhead separations (Fig. 5e). We concluded that a dynein dimer experiences intramolecular tension through its linker 
a
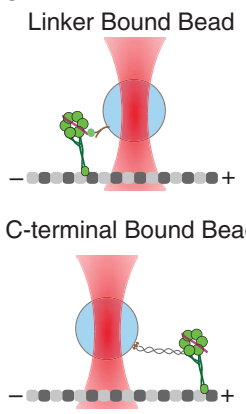

C-terminal Bound Bead

b

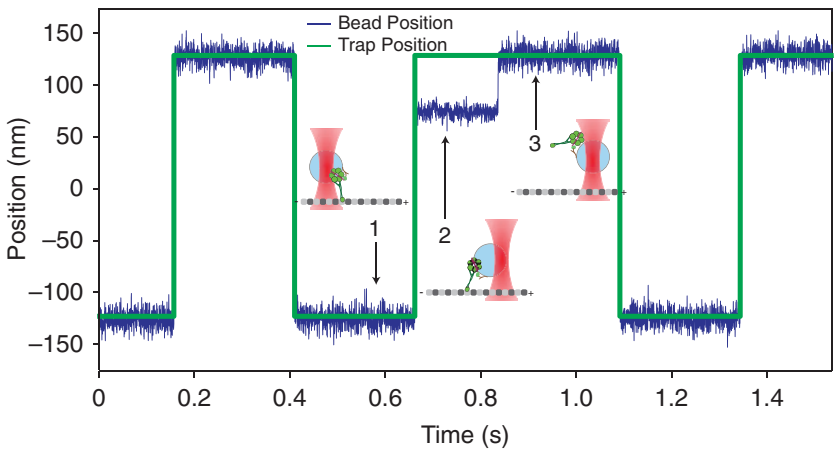

C

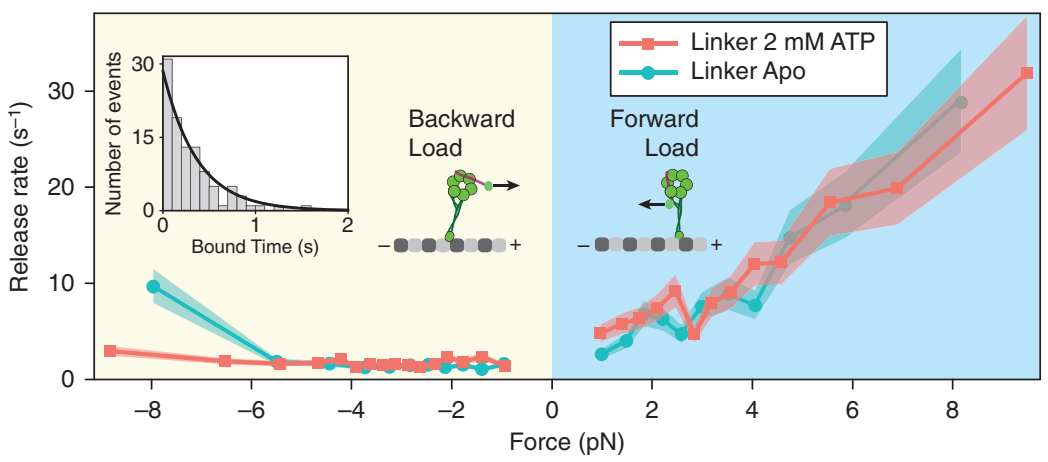

d

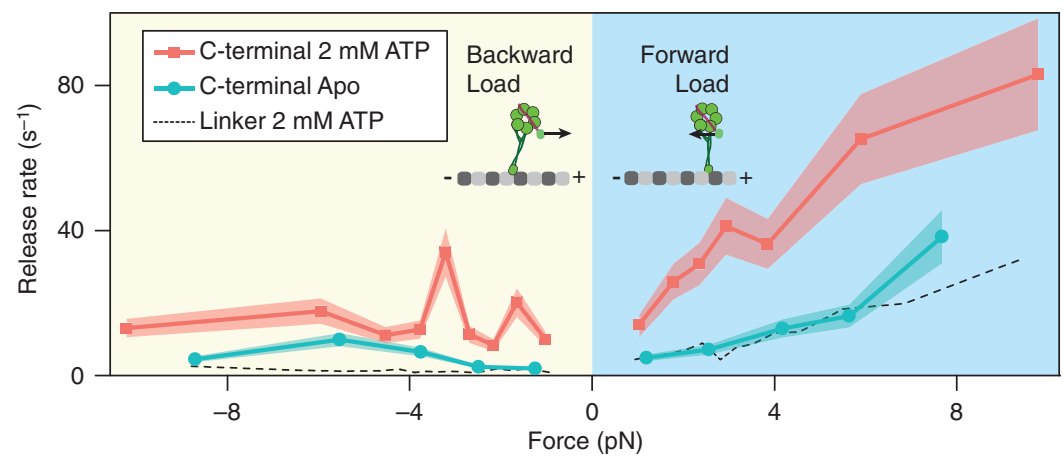

Figure 4 | Tension on the linker inhibits nucleotide-dependent release of dynein from MTs. (a) Dynein monomers are attached to a polystyrene bead either through the $\mathrm{N}$ terminus of the linker via a GFP-antibody linkage, or through the $\mathrm{C}$ terminus of the ring via a 74-bp long DNA tether (not to scale). (b) A representative trace showing bead position (blue) and trap position (green). (c) Force-dependent release rates of dynein monomers when the trapped bead is attached to the N-terminal linker domain. The distribution of the forward and backward release rates are unaffected by the presence (red, $n=3,003$ ) and absence (blue, $n=2,335$ ) of ATP. Shaded regions indicate $95 \%$ confidence intervals. Inset: a representative example of the distribution of measured MT-bound times at $0.94 \mathrm{pN}$ average force towards the MT minus-end at $1 \mathrm{mM}$ ATP. Black curve represents maximum likelihood fit to the dwell time histogram. (d) Force-dependent release rates when dynein is pulled through the $\mathrm{C}$ terminus. The rate is similar to the $\mathrm{N}$-terminal attachment case (dotted black line) in the absence of ATP (blue, $n=1,105$ ), but increases several fold in the presence of $2 \mathrm{mM}$ ATP (red, $n=1,652$ ).

domains at high interhead separations. Tension on the linker gates ATP-dependent stepping of the head from the MT.

A model for dynein motility. We combined our experimental results and developed a quantitative model for dynein motility. We envisioned that a head can release from the MT either through ATP binding or by tension (Fig. 6a). At low separations, the heads are fully uncoordinated. One of the heads binds ATP and takes a step with a rate of $8 \mathrm{~s}^{-1}$ (ref. 32). The other head serves as a tether to prevent release of the motor from $\mathrm{MT}^{18}$. At high interhead separations, tension on the linkers prohibits release of a head from MT through ATP binding (Fig. 6a). In this case, a head releases from MTs under tension and relieves the tension by stepping towards the tethered head.
We used reported step size $\mathrm{1}^{19,20}$ and ATPase rates ${ }^{30,33}$, as well as our optical trapping data, to test if our model could accurately capture the behaviour observed in native and mutant forms of yeast dynein. Release rates under tension were inferred from fits to the optical trap data (Fig. 5 and Supplementary Fig. 4). Monte Carlo simulations were used to generate stepping traces of a WT/WT homodimer. Simulated traces (Fig. 6b) reproduced uncoordinated stepping patterns, with a significant fraction $(33 \%)$ of steps in the backward direction ${ }^{18,32}$. At high interhead separations, the majority of the steps were taken by the rear head, as observed experimentally (Fig. 6c) ${ }^{18,19}$. The model estimated that the overall stepping rate per head is $7 \mathrm{~s}^{-1}$, in agreement with the MT-stimulated ATPase rates. The tensioninduced stepping pathway constitutes a significant fraction (32\%) of dynein stepping motion. 

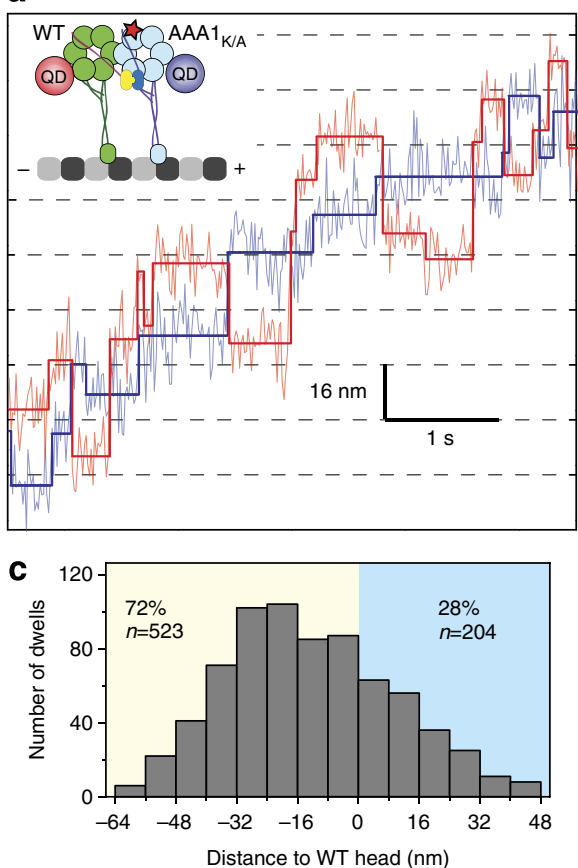

b
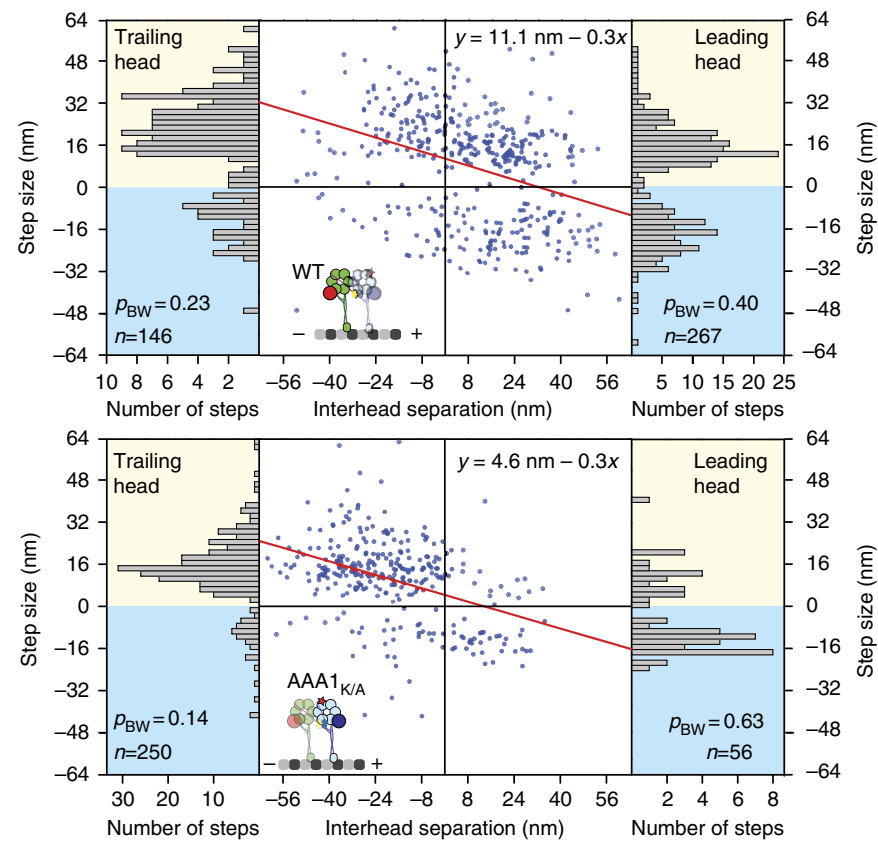

e

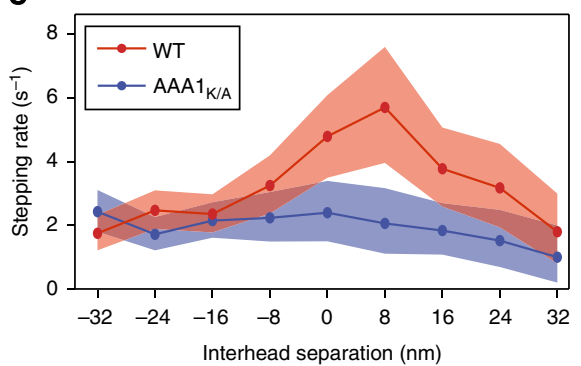

Figure 5 | Tension gating of a dynein head is observed at high interhead separations in a motile dimer. (a) A representative trace showing simultaneous tracking of $A A A 1_{K / A} / W T$ stepping properties with two colours of quantum dots at saturating $(1 \mathrm{mM})$ ATP. (b) The scatter plots represent the step sizes of WT (top) and $A A A 1_{K / A}$ (bottom) heads as a function of interhead separation. Interhead separation is positive when the stepping head is in the lead. Slope and $y$ intercept of the linear fit (red line) reveal the change in step size per nm extension between the heads and the net bias to move towards the minus-end, respectively. Both heads take smaller steps when they release the MT in the leading position, similar to native dynein. The AAA1 $1_{K / A}$ head takes mostly backward steps when stepping from the leading position (probability of backward stepping, $p_{\mathrm{BW}}=0.63$ ). (c) The $A A A 1_{K / A}$ head remains in the trailing position $72 \%$ of the time. Distance to the WT head is positive when the AAA1 $/$ /A head is in the lead. (d) Histogram of the dwell time between steps of the WT (left) or AAA1 $1_{K / A}$ (right) head, independent of the action of the other head, with exponential fit $( \pm 95 \% \mathrm{Cl}$ ). (e) Stepping rates of the WT and $A A A 1_{K / A}$ heads as a function of interhead separation (shaded region is the $95 \% \mathrm{Cl}$ ). The WT head steps more frequently when it is positioned close to the $A A A 1_{K / A}$ head, and is otherwise as a similar rate to the $A A A 1_{K / A}$ head, indicating that the WT head is gated at high interhead separations.

We also tested whether our model could reproduce the observed speeds of dynein motility in response to ATPase mutations and altered dimerization geometry. The stiffness of the linkage between the dynein heads remains unknown; therefore the tension between the heads cannot be directly inferred from the interhead separation. Therefore, we tested a wide range of values for linker stiffness (Supplementary Fig. 5). In simulations of these mutants, optical trap data with $\mathrm{C}$ - and N-terminal attachment geometries were used to calculate the tension-induced release rates of FLH and BLH, respectively (Supplementary Fig. 4). In the model, the FLH and $A A A 1_{E / Q}-$ mutant heads release from the MT through ATP binding, but do not undergo a powerstroke (Supplementary Fig. 3b).

We found that the stiffness of $1 \mathrm{pN}$ per 12 -nm extension of the linker agreed most closely with the experimental results (Fig. 6d and Supplementary Fig. 5). The velocities of simulated motors were within $\pm 15 \%$ of the measured velocities of mutant heterodimers. This number estimates that, at the highest interhead separations $(\sim 36 \mathrm{~nm})$, the heads experience 3-pN tension, equivalent to maximal force production of a single dynein motor (manuscript in preparation). The results show that the tensioninduced gating mechanism explains the stepping properties of dynein motility. See Methods for a detailed description of the parameters used in the model.

\section{Discussion}

Previous studies have shown that individual dynein motor domains are incapable of processive movement and that dimerization restores processivity ${ }^{32}$. However, it remained unclear whether dynein motility specifically requires both monomers to be catalytically active, dimerized through their $\mathrm{N}$-termini, communicating their nucleotide cycles and mechanically coupling their stepping motions with each other. 

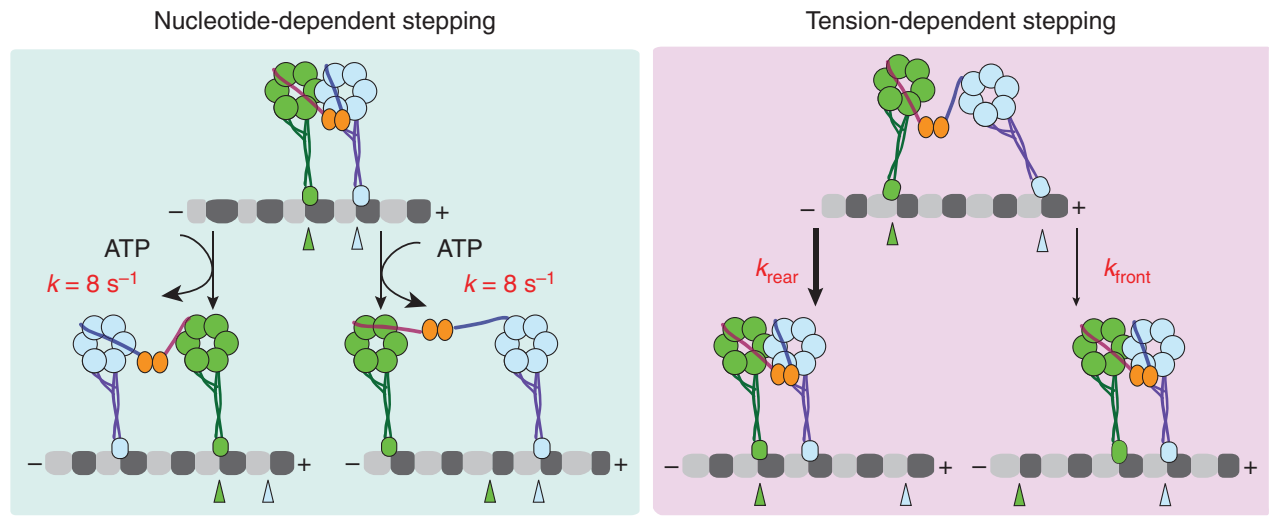

b

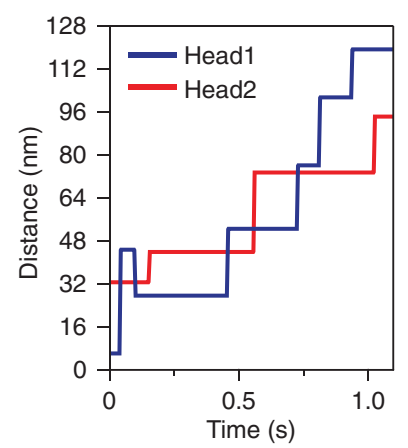

C

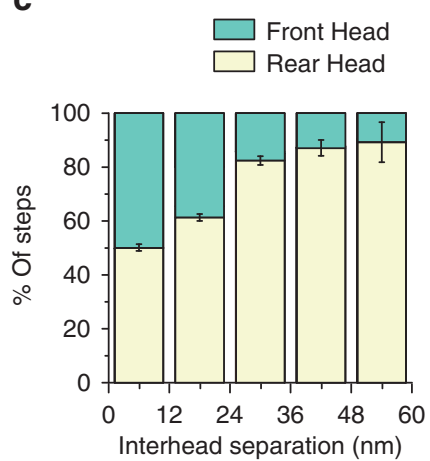

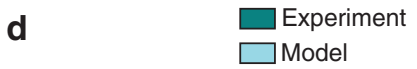

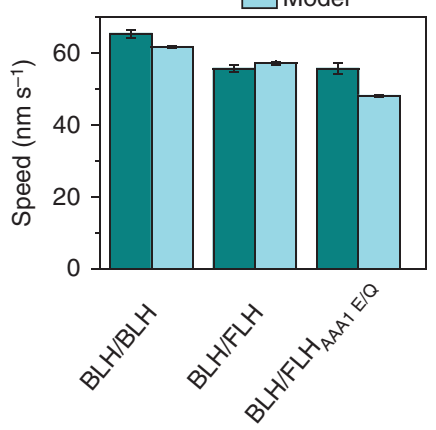

Figure 6 | A model for dynein motility. (a) Two proposed mechanisms of dynein stepping. (Left) when the heads are close to each other, either head can release the MT and step forward upon ATP binding. (Right) When the heads are far apart, tension on the linker prevents ATP-dependent MT release, and the asymmetry of the release rates under tension favors the trailing head to take a step. (b) A representative Monte Carlo simulation of dynein motility shows stepping of the two head domains (blue and red). (c) The trailing head is more likely to take a step in simulated traces as the interhead separation increases. The data shown are the average of 200 simulations ( \pm s.d.). (d) The average velocity of 200 100-s simulated traces ( \pm s.e.m.) agrees well with measured velocities for various dynein mutants ( \pm s.e.m., $n>100$ ). The results of the model are within $\pm 15 \%$ of the experimental data.

We engineered dynein's motile properties in predictable ways, which allowed careful examination of structural and catalytic requirements of dynein processivity. Processivity does not require two catalytically active motor domains. Altering the ATPase activity of a single monomer in dynein heterodimers revealed a strikingly different phenotype from identical mutations to homodimeric constructs ${ }^{29,30}$. A hydrolysis-deficient AAA1 mutant heterodimer moved at near-WT velocities. These results differ significantly from similar measurements on a kinesin heterodimer carrying a single ATP hydrolysis ${ }^{41}$ or binding ${ }^{42}$ mutation, which moves 10-20-fold slower than native kinesin. Consistent with the ability of dynein to move without tight interhead coordination, a single force-generating head is able to drive motility nearly as fast as if both heads retained full ATPase activity, provided its inactive partner has moderate affinity for the MT.

Dynein's unique domain architecture has made it possible to isolate the MT-binding interface from its catalytic core. We showed that N-terminal dimerization is not essential for processive motion and alternative dimerization geometries can achieve increased processivity with a minor reduction in motor velocity. The entire AAA + ring and linker domains of one head can be replaced with an inert protein retaining the MTBD and part of the stalk. Therefore, dynein motility does not require both $\mathrm{AAA}+$ ring domains, and the partner motor does not need to cycle between weakly and tightly MT-bound states. These results raise the possibility that synthetic processive motors may be engineered from a single polypeptide containing one force- producing head and two MT attachment sites. Our ringless dynein heterodimer also excludes direct mechanical or allosteric interactions between the two $\mathrm{AAA}+$ rings as a required mechanism for processivity or force generation.

How could dynein walk processively in the absence of interhead communication? We envision that processivity could arise if the duty ratio of a head is sufficiently high $(\sim 0.9)$ to allow the motor to take 100 steps (the average run length is $\sim 1 \mu \mathrm{m}$ and the average step size is $\sim 10 \mathrm{~nm}$ ) before dissociation ${ }^{18}$. The duty ratio of Dictyostelium dynein was estimated to be a minimum of 0.6 by the filament gliding assays ${ }^{36}$. Consistent with this idea, we observed that the processivity of engineered dynein heterodimers is correlated with the MT affinity of the mutant head.

A swinging motion of the linker domain was shown to drive the MT gliding ability of dynein monomers ${ }^{32,43}$. Engineering of a processive dynein dimer with mechanically distinct monomers allowed us to investigate in depth the function of the linker in dynein's stepping mechanism. By selectively disrupting the linker swing in FLH and BLH mutants, we present strong evidence that the linker powers the motility of a dynein dimer and its $\mathrm{N}$ terminus must be tethered to a second MT-binding site to generate an efficient powerstroke.

In addition to its function as a mechanical element, the linker is involved in coupling the nucleotide and mechanical states of the motor domain. Reducing the linker length ${ }^{32}$ or mutating the residues on AAA5 that make contact with the linker ${ }^{8}$ eliminate most of the MT-stimulated ATPase activity, indicating that contacts between the linker and the ring are essential for ATPase 
activity. Our observation that force on the linker influences dynein's ability to release from MT in a nucleotide-dependent manner further highlights the connection between MT affinity and linker conformation ${ }^{13}$. Tension on the linker may disrupt these connections and force the linker to attain a fixed conformation relative to the ring. It is also possible that tension on the linker decouples MT affinity from the ATP hydrolysis cycle, such that ATPase activity remains unabated, but MT affinity is not controlled by the nucleotide state. In this case, dynein monomers would be expected to show similar MT-release rates when pulled through the linker, independent of any ATPase mutations in the ring. However, we observed that $\mathrm{AAAl}_{\mathrm{E} / \mathrm{Q}}$ monomers have higher release rates than WT monomers when force is applied to the linker domain, implying that the nucleotide state is still coordinated with MT affinity (Supplementary Fig. 3b,c). Long-range communication between the catalytic sites and the MTBD can be mediated by a coiled-coil segment extending from AAA5 (refs 44,45). Since the linker runs across the opposite side of the ring ${ }^{7,8}$, it is unlikely that the linker orientation directly affects MT affinity. Instead, we propose that linker configuration is coupled to the catalytic activity of the ring, and indirectly affects the MT affinity.

In cells, dynein motor activity is regulated by several associated proteins including the dynactin complex, LIS1, and NUDEL ${ }^{46}$. Lis1 forms a dimer, and directly interacts with both motor domains. Lis1 binding induces a persistent high-affinity MTbound state and slows down dynein motility ${ }^{47,48}$. Our results describing how dynein motility responds to perturbations to one of its two motor domains show that for a dynein dimer to be fully inhibited each monomer must be bound to an inactivating factor. This would alter the response of dynein to cytosolic concentrations of regulatory proteins and allows tuning of dynein speed and processivity at different levels. Indeed, monomeric Lis1 is able to inhibit dynein motility, albeit at significantly higher concentrations than that of Lis1 dimer ${ }^{48}$.

During yeast cell division, multiple dynein motors bound to the cell periphery pull MTs in opposite directions across the cell. Proper segregation of nuclei requires dynein motors to remain bound to MTs under high tension. We showed that the dynein MTBD remains attached to MTs under plus-end-directed forces, even in the absence of Lis1. Tension on the linker domain also induces a prolonged MT-bound state by inactivating nucleotidedependent release from MT. This mechanism may contribute to dynein's ability to move large intracellular cargos and anchor MTs to the cell cortex under high tension.

\section{Methods}

Construct design and yeast cloning. An $\mathrm{N}$-terminal truncated $S$. cerevisiae cytoplasmic dynein gene (DYN1) encoding amino acids 1219-4093 (predicted molecular weight $331 \mathrm{kD}$, referred to as Dyn) was used as a template for mutagenesis $^{32}$. Dyn was artificially dimerized through an N-terminal GST tag (GSTDyn). An N-terminal GFP fusion protein of GST-Dyn (GFP-GST-Dyn) has been shown to move at similar velocity and processivity to that of native yeast dynein ${ }^{32}$.

Heterodimeric dynein constructs were obtained by replacing the GST tag of GST-Dyn with an FRB tag on one monomer (FRB-Dyn) and an FKBP12 tag on the other monomer (FKBP12-Dyn). FRB and FKBP12 form a heterodimer in the presence of the small molecule rapamycin ${ }^{31}$. A C-terminal HaloTag (Promega) was used to fluorescently label dynein.

FRB-Dyn constructs carrying ATPase mutations, $\mathrm{AAA}_{\mathrm{E} / \mathrm{Q}}(\mathrm{E} 1849 \mathrm{Q}), \mathrm{AAA} 3_{\mathrm{E} / \mathrm{Q}}$ (E2488Q) and monomeric Dyn $\mathrm{AAA}_{\mathrm{K} / \mathrm{A}}(\mathrm{K} 1802 \mathrm{~A}), \mathrm{AAA}_{\mathrm{K} / \mathrm{A}}(\mathrm{K} 2424 \mathrm{~A})$ were a generous gift of Ronald D. Vale (UCSF). An N-terminal FRB domain was added to the monomeric K/A mutants. FLH constructs were obtained by inserting an FRB or FKBP12 heterodimerization tag to the $\mathrm{C}$ terminus of a Dyn construct carrying the appropriate ATPase mutations ${ }^{32}$. Assays on FRB-BLH dimerized to FKBP12-FLH or with FKBP12-BLH dimerized to FRB-FLH did not show a large difference in motor speed verifying that the speed measurements are not affected by which tag is introduced to $\mathrm{C}$ and $\mathrm{N}$ terminus of the motor.

Chimeric constructs which encode a monomeric Thermus thermophilus SRS and the dynein MTBD at different coiled-coil length and registry were generously provided by Ian Gibbons and Andrew P. Carter ${ }^{34}$. A $4 \times(\mathrm{GS})$-FKBP12 tag was inserted to the $\mathrm{C}$ terminus of SRS. For optical trapping experiments, an enhanced GFP gene was added in place of FKBP12. The SRS construct was additionally tagged with an N-terminal HaloTag for fluorescent labelling. Supplementary Table 1 contains all of the constructs used in this study.

Protein expression, purification and labelling. Dynein proteins were expressed in yeast and purified as described ${ }^{32}$. Purified protein was stored in dynein loading buffer (DLB; $30 \mathrm{mM}$ HEPES pH 7.2, $2 \mathrm{mM} \mathrm{MgCl}_{2}, 1 \mathrm{mM}$ EGTA, $10 \%$ glycerol). Expression and purification of SRS-MTBD mutants were carried out in Escherichia coli, as described ${ }^{34}$. For fluorescent tracking experiments, BLH heads containing a C-terminal HaloTag were labelled with $10 \mu \mathrm{M}$ tetramethylrhodamine (TMR) HaloTag ligand (Promega) for $1 \mathrm{~h}$ on ice during the protein preparation, before washing of the Immunoglobulin G beads (GE Healthcare).

SRS chimera MT co-sedimentation assay. The SRS chimera MT co-sedimentation assay was carried out in DLB as described ${ }^{34}$.

Microscope. Single-molecule motility assays were performed on a custom-built objective-type total internal reflection fluorescence microscope, equipped with an inverted microscope body (Nikon Ti-Eclipse) with perfect focusing system, 1.45 NA $60 \times$ microscope objective (Nikon, TIRF Plan Apochromat). The sample was illuminated with 488 and $532 \mathrm{~nm}$ solid-state lasers (Coherent) to image GFP and TMR, respectively. BLH-TMR was imaged to record the speed and run length data except in Supplementary Movie 1 in which SRS-TMR is imaged. FLH-GFP was imaged to confirm motility of the FLH (data not shown). For velocity assays, GSTDyn motility was recorded with a 2-s exposure time under $5.1 \mathrm{~mW} 532$-nm laser exposure. For run length assays, GST-Dyn motility was recorded under $1.9 \mathrm{~mW}$ of 532-nm illumination. Laser power and the image acquisition rate were adjusted for other constructs to keep the average distance travelled by motors $(\sim 100 \mathrm{~nm}$ per image) and the bleaching decay rate of TMR (0.004 per image) constant. Run length assays were done in buffer containing a final concentration of $75 \mathrm{mM} \mathrm{K}^{+}$ Emitted photons were detected by an electron-multiplied charge-coupled device camera (Andor Ixon, $512 \times 512$ pixels, $16 \mu \mathrm{m}$ pixel size). The image was magnified by a tube lens to obtain $129.3 \mathrm{~nm}$ effective pixel size, calibrated with a reticle containing 100 lines per $\mathrm{mm}$.

Single-molecule motility assays. Motility assays were performed as described ${ }^{32}$ Sea urchin axonemes were immobilized on a glass coverslip in a flow chamber constructed with double-sided tape. The chamber was washed with $50 \mu$ l of DLB followed by $50 \mu \mathrm{l}$ of DLB supplemented with $1 \mathrm{mg} \mathrm{ml}^{-1}$ casein, $2 \mathrm{mM}$ DTT (DLBC). In total, $200 \mathrm{pM}$ dynein was then perfused into the chamber in DLBC and allowed to bind to MT for $1 \mathrm{~min}$. The flow cell was then washed with $100 \mu \mathrm{l}$ of DLBC and $20 \mu \mathrm{l}$ of imaging buffer (DLBC with $1 \% \beta$-mercaptoethanol, $2 \mathrm{mM}$ ATP and an oxygen scavenging system consisting of $25 \mathrm{mM}$ protocatechuic acid (PCA), $0.35 \mathrm{mg} \mathrm{ml}^{-1}$ protocatechuate-3,4-dioxygenase, $0.6 \mathrm{mM}^{\text {Trolox }}{ }^{49}$ ).

For assays of FRB-FKBP12 heterodimers, $1 \mu \mathrm{l}$ of $600 \mathrm{nM}$ rapamycin and a total volume of $2 \mu \mathrm{l}$ of equimolar amounts of FRB- and FKBP12-tagged monomers were mixed and incubated in DLB for $10 \mathrm{~min}$ at room temperature. These constructs were then further diluted in DLBC before introducing the motor to sample chamber. In total, $100 \mathrm{nM}$ rapamycin was added to all assay buffers to maintain the dimerization. We are confident that the motility observed was due to

heterodimerization because: (1) under the motor concentrations used, we did not observe motility in the presence of only one type of the FRB- or FKBP12-tagged monomers; (2) processive motility of FRB- and FKBP12-tagged monomers was also not observed in the absence of rapamycin; (3) selective ATPase mutations on $\mathrm{BLH}$ and FLH resulted in dramatic changes in dynein motility, depending on which head carried the mutation, which would not be possible if the motors observed were not heterodimers; (4) fluorescently labelled SRS chimeras are observed to be motile when heterodimerized with unlabelled dynein monomers (Supplementary Movie 1).

Multicolour tracking of $\mathrm{AAA}_{\mathrm{K} / \mathrm{A}} / \mathrm{WT}$ was performed at $1 \mathrm{mM}$ ATP and movies were recorded at $15 \mathrm{~ms}$ frame rate. Quantum dot labelling, imaging and data analysis of the assay were performed as described ${ }^{18}$. Fluorescent spots of individual quantum dots were localized with a two-dimensional Gaussian tracking algorithm. Trajectories of dyneins were fitted by a custom-written step finder algorithm, based on Schwartz Information Criterion ${ }^{50}$.

Optical trapping assay. A custom-built optical trap consisting of a 2-W 1,064-nm continuous wave laser (Coherent), a Nikon Ti-Eclipse microscope body and a Nikon $100 \times 1.49$ NA Plan-Apo objective was used to study force-dependent release of dynein monomers. To apply forces through the N-terminal linker of dynein, GFP-tagged dynein monomers were diluted in DLBC and incubated with carboxylated polystyrene beads $(0.86 \mu \mathrm{m}$ diameter, Invitrogen) coated with a rabbit polyclonal anti-GFP antibody (Covance). Antibody coating of the beads was carried out by EDC-NHS crosslinking (Pierce) ${ }^{40}$. The motor-bead mixture was then diluted tenfold in DLBC with $5 \mathrm{mg} \mathrm{ml}^{-1}$ casein, the PCA/PCD oxygen scavenging system and $2 \mathrm{mM}$ ATP. For the apo condition, ATP was not added to the motorbead mixture, resulting in residual ATP concentrations of $\sim 40 \mathrm{nM}$, well below 
dynein's $K_{\mathrm{M} \text {, АтP }} \sim 26 \mu \mathrm{M}$ (ref. 29 ). The final bead concentration was $0.1 \%$ weight/volume. To record data from single monomers, motors were diluted to a level where we observed $5-15 \%$ binding probability during the oscillation of the beads. In total, $>90 \%$ of the binding events terminated with a single release step, indicative of binding of a single dynein monomer. At tenfold higher motor concentrations, significant increase in multiple release events was observed (data not shown), while no events were observed in the absence of motor.

To apply forces through the C-terminal ring domain, dynein monomers with a C-terminal Halo tag were labelled with a short (74 bp) double-stranded DNA strand. Two complimentary DNA strands, one with a $5^{\prime}$-biotin modification (5'-Biotin-TTCGGTCAATACCCGGCGCAGAGCGCTCAGGCGCGAG GTCAACAGAGGGCGGAGGGTGGGCCAGCGCGACCCCG-3'), the other with a $5^{\prime}$-amine modification (5'-AmMC6-GTGTCGGGGTCGCGCTGGCC CACCCTCCGCCCTCTG TTGACCTCGCGCCTGAGCGCTCTGCGCC GGGTATTGAC-3') (IDT) were hybridized by combining $30 \mu \mathrm{l}$ of each strand from $100 \mu \mathrm{M}$ stock with $20 \mu \mathrm{l}$ of DNA buffer $\left(80 \mathrm{mM} \mathrm{NaHCO}_{3} \mathrm{pH} 8.4,200 \mathrm{mM}\right.$ $\mathrm{KCl}, 5 \mathrm{mM} \mathrm{MgCl}$ ), heating at $90^{\circ} \mathrm{C}$ for $20 \mathrm{~min}$, cooling to $25^{\circ} \mathrm{C}$ for $40 \mathrm{~min}$. The DNA mixture was then reacted with $160 \mu \mathrm{M}$ HaloTag NHS ligand (Promega) for $6 \mathrm{~h}$ at room temperature and the reaction quenched with $1 \mu \mathrm{l}$ of $1 \mathrm{M}$ glycine, $\mathrm{pH}$ 8.4. DNA was then desalted through G-25 spin columns into DNA buffer. Dynein $(\sim 400 \mu \mathrm{l})$ was then labelled with the purified HaloTag-DNA-biotin for $6 \mathrm{~h}$ on ice, and excess DNA was removed through microtubule bind and release purification ${ }^{32}$. This dynein-DNA-biotin complex was then incubated with streptavidin-coated polystyrene beads $(0.86 \mu \mathrm{m}$ diameter, Invitrogen $)$, as described above. The motorbead mixture was then diluted in the imaging buffer.

Cy5-labelled axonemes were adsorbed to the surface of a flow cell before addition of the bead/dynein mixture. The sample was excited with $633 \mathrm{~nm} \mathrm{HeNe}$ laser (Melles Griot) and axonemes were visualized with a CCD camera. The trapping beam was steered by two computer-controlled acousto-optical deflectors (AA Electronics) to capture and position floating monodisperse beads. Trap stiffness was calibrated for each sample by fitting the windowed power spectrum of a bead trapped $3 \mu \mathrm{m}$ above the surface of the coverslip to a Lorentzian curve $\mathrm{e}^{51}$. The microspheres were trapped by a $\sim 50-\mathrm{mW} 1,064-\mathrm{nm}$ laser beam to achieve a spring constant of $\sim 0.05 \mathrm{pN} \mathrm{nm}^{-1}$. A position-sensitive detector (PSD) was located at the back focal plane to detect microsphere displacement. The PSD data were recorded at $20 \mathrm{kHz}$ for calibration and $5 \mathrm{kHz}$ for data acquisition. The response of the PSD was calibrated in each sample by rapidly scanning the laser across a trapped bead in both $x$ and $y$ directions using the acousto-optical deflectors and fitting the resulting curve to a cubic polynomial. This calibration was repeated once at the surface and once $3 \mu \mathrm{m}$ into the solution to avoid systematic errors in either experimental data or stiffness calibrations.

Trapped beads were positioned over a Cy5-labelled axoneme and oscillated between two positions ( $\pm 125 \mathrm{~nm}$ ) along the long axis of the axoneme. Bead-trap separation was monitored in real time to prevent trap oscillations during a binding event. The trap was held steady for $0.25 \mathrm{~s}$ after the bead returned to the trap centre, and then moved to the opposite position. Microtubule polarity was determined by imaging Oregon green-labelled kinesin motors, which decorate the plus-end of MTs. GFP-labelled kinesin was used to determine MT polarity in trapping experiments on DNA-tethered dynein monomers, which lack GFP. Strong (three to tenfold) asymmetry was observed in the release rate of both linker- and C-terminal-bound dynein monomers in the presence of ATP (all of 11 axonemes tested, data not shown). The rest of the data were collected by assigning the microtubule polarity, based on the asymmetry in the plus- and minus-end-directed release rates of dynein monomers.

Data analysis. The kymographs of GFP-tagged dyneins were made in ImageJ. For the run length analysis, we chose molecules that moved at least 4 pixels $(\sim 500 \mathrm{~nm})$, began their runs at least $5 \mu \mathrm{m}$ away from the minus-end of MTs and remain fluorescent for at least 50 frames before the end of the movie. Since the average distance travelled by a motor before photobleaching $(25 \mu \mathrm{m})$ is much greater than the measured average run length $(0.8-2.2 \mu \mathrm{m})$, we have not included a correction for photobleaching in our analysis. Such a correction would result in an increase of run length of less than $5 \%$, within the error of our measurement. The mean run length was calculated by maximum likelihood estimation of the exponential decay constant and stated errors are the $95 \%$ confidence intervals.

In optical trap assays, single-step MT-release events were analysed and rare multiple-step release events were discarded from the data analysis. Force-induced MT-release data were fit with a custom step-finding algorithm. Events showing a dwell time of greater than $2.5 \mathrm{~ms}$ were evaluated visually to confirm the binding and release of a single monomer. The applied force to the motor was calculated by trap stiffness and the bead-trap separation vector. Data consisting of applied force and dwell time were sorted by force and binned every 100 data points. The dwell times in each bin were fit to a single exponential decay (see Fig. 4c, inset), and resulting rate constant was plotted with the average force of all the data points in the bin. Errors are the 95\% confidence intervals. Changing the bin size did not significantly affect the results (not shown).

Stepping rate versus interhead separation (Fig. 5d) was calculated by taking the number of steps each motor took in a given range of interhead separation (8-nm wide bins from -36 to $36 \mathrm{~nm}$ ) and dividing by the total amount of time the motor spent in that range of separation. In total, $95 \%$ confidence intervals were calculated by bootstrapping. Larger interhead separations were not considered due to the rarity of steps, $<10$ per bin, preventing accurate determination of stepping rate. In a WT/WT motor, the rates for each motor fall within the $95 \%$ confidence interval of each other at all interhead separations (data not shown).

Computational model. Monte Carlo simulations were run to generate stepping traces of a WT dynein dimer. The model incorporates parameters from bulk ATPase ${ }^{29,32}$, optical trap and stepping measurements ${ }^{18,19}$ of yeast dynein (see below). The model assumes ATP-dependent steps can occur when the interhead separation is less than $20 \mathrm{~nm}$. The WT head can step in an ATP-dependent manner with a rate $k_{1}=8 \mathrm{~s}^{-1}$ based on bulk ATPase measurements per head ${ }^{29,32}$. The stepping head rebinds the MT at a position randomly chosen from a Gaussian distribution with a mean $8 \pm 16 \mathrm{~nm}$ ( \pm s.d.) towards the minus-end from its partner head. The 8-nm minus-end-directed bias in step size is provided by the ATP-dependent linker swing mechanism ${ }^{9}$.

$\mathrm{FLH}_{\mathrm{WT}}$ and $\mathrm{AAA} 1_{\mathrm{E} / \mathrm{Q}}$ mutants, which lack the ability to generate a powerstroke, undergo non-productive ATP-dependent stepping with a rate $k_{2} \sim 20 \mathrm{~s}^{-1}$, measured from extrapolation to the force-induced release data at $0 \mathrm{pN}$ force at $1 \mathrm{mM}$ ATP (Supplementary Fig. 4). The stepping head rebinds at a position randomly chosen from a Gaussian distribution centered at its partner head with $0 \pm 16 \mathrm{~nm}$ (s.d.) step size.

Tension-dependent steps occur when the interhead separation is greater than $12 \mathrm{~nm}$. In this mode of stepping, the leading head steps backwards with a constant rate of $k_{3}$, determined from the force-induced release data (Fig. 4 and

Supplementary Fig. 4). The trailing head steps with a rate $k_{4}=m x$, where $x$ is the interhead separation in $\mathrm{nm}$. $m$ was calculated from linear fits to the force-induced release data (Fig. 4 and Supplementary Fig. 4). The release rates of FLH and BLH under tension were calculated from force-dependent release data on motors pulled from their $\mathrm{C}$ terminus and linker, respectively. Tension-induced stepping rate increases at higher interhead separations due to increase in intramolecular tension. Fixing the release rate to be equal to the rate measured for a $1-3 \mathrm{pN}$ bin of force data at all interhead separations did not significantly affect the speeds of any of the mutants (data not shown). After a tension-based step, the motor rebinds the MT at a position randomly chosen from a Gaussian distribution, $8 \pm 16 \mathrm{~nm}$ from its partner head in the direction of the initial position of the stepping head. When the heads are between 12 and $20 \mathrm{~nm}$ apart, both tension-dependent and -independent steps are possible. At separations higher than $20 \mathrm{~nm}$, the ATP-dependent stepping mechanism is abolished due to the increased tension on the linker domain (Fig. 4c,d). Excluding tension-independent steps in this state did not significantly affect the results.

Monte Carlo simulations. The Python code used in the simulations is available at http://physics.berkeley.edu/research/yildiz/SubPages/code_repository.html.

For each dwell of the motor, the tension-based rate for the trailing head was recalculated according to the fit line of the optical trap data, and the assumed stiffness of the linkage between monomers $\left(1 / 12 \mathrm{pN} \mathrm{nm}^{-1}\right.$ ) (Supplementary Fig. 5). Other rates used in the model are shown in Supplementary Table 2. For each mutant simulated, 200 simulations were run for $100 \mathrm{~s}$ with a time step of $0.005 \mathrm{~s}$ to estimate the speed of each construct. In simulations of mutant dyneins, $\mathrm{C}$ - and N-terminal pulling data were used to calculate the force-induced release rates of FLH and BLH, respectively.

\section{References}

1. Vallee, R. B., Williams, J. C., Varma, D. \& Barnhart, L. E. Dynein: an ancient motor protein involved in multiple modes of transport. J. Neurobiol. 58, 189-200 (2004).

2. Carminati, J. L. \& Stearns, T. Microtubules orient the mitotic spindle in yeast through dynein-dependent interactions with the cell cortex. J. Cell Biol. 138, 629-641 (1997).

3. Morales-Mulia, S. \& Scholey, J. M. Spindle pole organization in Drosophila S2 cells by dynein, abnormal spindle protein (Asp), and KLP10A. Mol. Biol. Cell 16, 3176-3186 (2005).

4. Griffis, E. R., Stuurman, N. \& Vale, R. D. Spindly, a novel protein essential for silencing the spindle assembly checkpoint, recruits dynein to the kinetochore. J. Cell Biol. 177, 1005-1015 (2007).

5. Hafezparast, M. et al. Mutations in dynein link motor neuron degeneration to defects in retrograde transport. Science 300, 808-812 (2003).

6. Gerdes, J. M. \& Katsanis, N. Microtubule transport defects in neurological and ciliary disease. Cell Mol. Life Sci. 62, 1556-1570 (2005).

7. Kon, T. et al. The 2.8 A crystal structure of the dynein motor domain. Nature 484, 345-350 (2012).

8. Schmidt, H., Gleave, E. S. \& Carter, A. P. Insights into dynein motor domain function from a 3.3-A crystal structure. Nat. Struct. Mol. Biol. 19, 492-497 (2012).

9. Roberts, A. J. et al. AAA + Ring and linker swing mechanism in the dynein motor. Cell 136, 485-495 (2009).

10. Gee, M. A., Heuser, J. E. \& Vallee, R. B. An extended microtubule-binding structure within the dynein motor domain. Nature 390, 636-639 (1997). 
11. Burgess, S. A., Walker, M. L., Sakakibara, H., Knight, P. J. \& Oiwa, K. Dynein structure and power stroke. Nature 421, 715-718 (2003).

12. Kon, T., Mogami, T., Ohkura, R., Nishiura, M. \& Sutoh, K. ATP hydrolysis cycle-dependent tail motions in cytoplasmic dynein. Nat. Struct. Mol. Biol. 12, 513-519 (2005).

13. Imamula, K., Kon, T., Ohkura, R. \& Sutoh, K. The coordination of cyclic microtubule association/dissociation and tail swing of cytoplasmic dynein. Proc. Natl Acad. Sci. USA 104, 16134-16139 (2007).

14. Yildiz, A. et al. Myosin V walks hand-over-hand: single fluorophore imaging with 1.5-nm localization. Science 300, 2061-2065 (2003).

15. Asbury, C. L., Fehr, A. N. \& Block, S. M. Kinesin moves by an asymmetric hand-over-hand mechanism. Science 302, 2130-2134 (2003).

16. Yildiz, A., Tomishige, M., Vale, R. D. \& Selvin, P. R. Kinesin walks hand-overhand. Science 303, 676-678 (2004).

17. Kodera, N., Yamamoto, D., Ishikawa, R. \& Ando, T. Video imaging of walking myosin V by high-speed atomic force microscopy. Nature 468, 72-76 (2010).

18. DeWitt, M. A., Chang, A. Y., Combs, P. A. \& Yildiz, A. Cytoplasmic dynein moves through uncoordinated stepping of the AAA + ring domains. Science 335, 221-225 (2012).

19. Qiu, W. et al. Dynein achieves processive motion using both stochastic and coordinated stepping. Nat. Struct. Mol. Biol. 19, 193-200 (2012).

20. Block, S. M. Kinesin motor mechanics: binding, stepping, tracking, gating, and limping. Biophys. J. 92, 2986-2995 (2007).

21. Hancock, W. O. \& Howard, J. Kinesin's processivity results from mechanical and chemical coordination between the ATP hydrolysis cycles of the two motor domains. Proc. Natl Acad. Sci. USA 96, 13147-13152 (1999).

22. Spudich, J. A. Molecular motors take tension in stride. Cell 126, 242-244 (2006).

23. Yildiz, A., Tomishige, M., Gennerich, A. \& Vale, R. D. Intramolecular strain coordinates kinesin stepping behavior along microtubules. Cell 134, 1030-1041 (2008).

24. Shastry, S. \& Hancock, W. O. Neck linker length determines the degree of processivity in kinesin-1 and kinesin-2 motors. Curr. Biol. 20, 939-943 (2010).

25. Dunn, A. R., Chuan, P., Bryant, Z. \& Spudich, J. A. Contribution of the myosin VI tail domain to processive stepping and intramolecular tension sensing. Proc. Natl Acad. Sci. USA 107, 7746-7750 (2010).

26. Klumpp, L. M., Hoenger, A. \& Gilbert, S. P. Kinesin's second step. Proc. Natl Acad. Sci. USA 101, 3444-3449 (2004).

27. Cashikar, A. G. et al. Defining a pathway of communication from the C-terminal peptide binding domain to the N-terminal ATPase domain in a AAA protein. Mol. Cell 9, 751-760 (2002).

28. Nicastro, D. et al. The molecular architecture of axonemes revealed by cryoelectron tomography. Science 313, 944-948 (2006).

29. Cho, C., Reck-Peterson, S. L. \& Vale, R. D. Regulatory ATPase sites of cytoplasmic dynein affect processivity and force generation. J. Biol. Chem. 283, 25839-25845 (2008)

30. Kon, T., Nishiura, M., Ohkura, R., Toyoshima, Y. Y. \& Sutoh, K. Distinct functions of nucleotide-binding/hydrolysis sites in the four AAA modules of cytoplasmic dynein. Biochemistry 43, 11266-11274 (2004).

31. Banaszynski, L. A., Liu, C. W. \& Wandless, T. J. Characterization of the FKBP.rapamycin.FRB ternary complex. J. Am. Chem. Soc. 127, 4715-4721 (2005).

32. Reck-Peterson, S. L. et al. Single-molecule analysis of dynein processivity and stepping behavior. Cell 126, 335-348 (2006).

33. Kon, T. et al. Helix sliding in the stalk coiled coil of dynein couples ATPase and microtubule binding. Nat. Struct. Mol. Biol. 16, 325-333 (2009).

34. Gibbons, I. R. et al. The affinity of the dynein microtubule-binding domain is modulated by the conformation of its coiled-coil stalk. J. Biol. Chem. 280, 23960-23965 (2005)

35. Carter, A. P. et al. Structure and functional role of dynein's microtubulebinding domain. Science 322, 1691-1695 (2008).

36. Shima, T., Imamula, K., Kon, T., Ohkura, R. \& Sutoh, K. Head-head coordination is required for the processive motion of cytoplasmic dynein, an AAA + molecular motor. J. Struct. Biol. 156, 182-189 (2006).
37. Gennerich, A. \& Vale, R. D. Walking the walk: how kinesin and dynein coordinate their steps. Curr. Opin. Cell Biol. 21, 59-67 (2009).

38. Capitanio, M. et al. Ultrafast force-clamp spectroscopy of single molecules reveals load dependence of myosin working stroke. Nat. Methods 9, 1013-1019 (2012).

39. Uemura, S. et al. Kinesin-microtubule binding depends on both nucleotide state and loading direction. Proc. Natl Acad. Sci. USA 99, 5977-5981 (2002).

40. Gennerich, A., Carter, A. P., Reck-Peterson, S. L. \& Vale, R. D. Force-induced bidirectional stepping of cytoplasmic dynein. Cell 131, 952-965 (2007).

41. Thoresen, T. \& Gelles, J. Processive movement by a kinesin heterodimer with an inactivating mutation in one head. Biochemistry 47, 9514-9521 (2008).

42. Kaseda, K., Higuchi, H. \& Hirose, K. Alternate fast and slow stepping of a heterodimeric kinesin molecule. Nat. Cell Biol. 5, 1079-1082 (2003).

43. Shima, T., Kon, T., Imamula, K., Ohkura, R. \& Sutoh, K. Two modes of microtubule sliding driven by cytoplasmic dynein. Proc. Natl Acad. Sci. USA 103, 17736-17740 (2006)

44. Carter, A. P., Cho, C., Jin, L. \& Vale, R. D. Crystal structure of the dynein motor domain. Science 331, 1159-1165 (2011).

45. Kon, T., Sutoh, K. \& Kurisu, G. X-ray structure of a functional full-length dynein motor domain. Nat. Struct. Mol. Biol. 18, 638-642 (2011).

46. Vallee, R. B., McKenney, R. J. \& Ori-McKenney, K. M. Multiple modes of cytoplasmic dynein regulation. Nat. Cell Biol. 14, 224-230 (2012).

47. McKenney, R. J., Vershinin, M., Kunwar, A., Vallee, R. B. \& Gross, S. P. LIS1 and NudE induce a persistent dynein force-producing state. Cell 141, 304-314 (2010).

48. Huang, J., Roberts, A. J., Leschziner, A. E. \& Reck-Peterson, S. L. Lis1 acts as a "clutch" between the ATPase and microtubule-binding domains of the dynein motor. Cell 150, 975-986 (2012).

49. Rasnik, I., McKinney, S. A. \& Ha, T. Nonblinking and long-lasting singlemolecule fluorescence imaging. Nat. Methods 3, 891-893 (2006).

50. Kalafut, B. \& Visscher, K. An objective, model-independent method for detection of non-uniform steps in noisy signals. Comput. Phys. Commun. 179, 716-723 (2008)

51. Visscher, K. \& Block, S. M. Versatile optical traps with feedback control. Methods Enzymol. 298, 460-489 (1998).

\section{Acknowledgements}

We thank the members of the Yildiz laboratory for helpful discussions and R. D. Vale and C. Cho (University of California, San Francisco), Andrew P. Carter (MRC, Cambridge) for providing yeast strains and plasmids. This work has been supported by NIH (GM094522 (A.Y.)), NSF CAREER Award (MCB-1055017 (A.Y.)), NSF Graduate Research Fellowship (DGE 1106400 (F.B.C. and V.B.)) and Burroughs Welcome Foundation (A.Y.)

\section{Author contributions}

F.B.C. and A.Y. designed experiments; F.B.C. and D.D.C. cloned, expressed, purified and characterized the protein; F.B.C., V.B. and T.B. performed optical trapping assays, F.B.C and Z.M.H. and A.Y.C. performed single molecule fluorescence assays, M.A.D. performed two-colour fluorescent tracking assays, and F.B.C. and A.Y. wrote the manuscript.

\section{Additional information}

Supplementary Information accompanies this paper at http://www.nature.com/ naturecommunications

Competing financial interests: The authors declare no competing financial interests.

Reprints and permission information is available online at http://npg.nature.com/ reprintsandpermissions/

How to cite this article: Cleary, F. B. et al. Tension on the linker gates the ATP-dependent release of dynein from microtubules. Nat. Commun. 5:4587 doi: $10.1038 /$ ncomms5587 (2014). 